PROCEEDINGS OF THE

AMERICAN MATHEMATICAL SOCIETY

Volume 140, Number 6, June 2012, Pages 1963-1972

S 0002-9939(2011)11079-7

Article electronically published on September 27, 2011

\title{
CONNECTIVITY OF JULIA SETS FOR WEIERSTRASS ELLIPTIC FUNCTIONS ON SQUARE LATTICES
}

\author{
JOSHUA J. CLEMONS
}

(Communicated by Bryna Kra)

\begin{abstract}
We show that the Julia set of a Weierstrass elliptic function on a square lattice is connected. The techniques used to prove this result are used to show a similar result for a related family of rational maps obtained from the Laurent series.
\end{abstract}

\section{INTRODUCTION}

It is a classical result that the Julia set of $z^{2}+c$ is connected if and only if the orbit of 0 is bounded. This could be restated as: "The Julia set of $z^{2}+c$ is connected if and only if each Fatou component contains at most one critical value." It is our goal in this paper to show that the Julia set of a Weierstrass elliptic function on a square lattice is connected by proving that each Fatou component contains at most one critical value. In certain specific cases the Julia set has been shown to be connected. These cases include when $\wp_{\Omega}$ has a real super-attracting fixed point [12] or a cycle of Siegel disks [14]. We complete the cases when $\wp_{\Omega}$ has attracting or parabolic periodic points. A result of $[5$ proves that all of these cases do indeed occur.

Using different techniques to show that critical values lie in distinct Fatou components, the Julia set of a Weierstrass elliptic on so-called triangular lattices has been shown to be connected [14, 13, 15. Our techniques are necessarily different due to the fact that one critical value is always a pole. Furthermore, the triangular lattice case has three different but symmetric (under rotations of $2 \pi / 3$ ) orbits of critical values, whereas the square case has one critical value which is a pole and the other two critical values map to the same value determining one free critical orbit. This results in at most one non-repelling cycle [4]. More generally, a Weierstrass elliptic function can have as many as three different non-repelling cycles of possibly different types.

This paper is setup as follows. In Section 2 we give the relevant technical details of Weierstrass elliptic functions. In particular, we discuss lattices, critical points, critical values, and mapping properties. Section 3 summarizes known results about

Received by the editors September 8, 2010 and, in revised form, January 24, 2011 and January $31,2011$.

2010 Mathematics Subject Classification. Primary 37F10, 54H20; Secondary 37F20 .

This is part of completed Ph.D. work at the University of North Carolina under the supervision of Jane Hawkins.

The author would like to thank the reviewer for the helpful suggestions that improved this paper. 
the Fatou and Julia sets of Weierstrass elliptic functions and their application to the square lattice setting. The main theorem is stated and proved in Section 4 . The last section applies the technique used in Section 4 to a related family of rational maps obtained from the Laurent series of the Weierstrass elliptic functions.

\section{LAtTices AND Weierstrass ElLiptic FUnCtions}

The Weierstrass $\wp$ function is considered to be the most basic of the family of elliptic functions. With that consideration we would like to study it in detail so that we may better understand other elliptic functions. Let $\mathbb{C}$ denote the complex plane, $\mathbb{C}^{*}=\mathbb{C} \backslash\{0\}$ and $\mathbb{C}_{\infty}=\mathbb{C} \cup\{\infty\}$ be the Riemann sphere.

Definition 2.1. A lattice $\Omega$ is an additive subgroup of $\mathbb{C}$ with two generators that is isomorphic to $\mathbb{Z}^{2}$ such that the generators $\omega_{1}, \omega_{2} \in \mathbb{C}^{*}$ are $\mathbb{R}$ linearly independent:

$$
\Omega=\left\{m \omega_{1}+n \omega_{2}: m, n \in \mathbb{Z}\right\} .
$$

We denote this by $\Omega=\left\langle\omega_{1}, \omega_{2}\right\rangle$.

The residue class of a point $z$ with respect to a lattice $\Omega$ is the set of values

$$
z+\Omega=\{z+\omega: \omega \in \Omega\},
$$

and a fundamental region is a simply connected region in $\mathbb{C}$ that contains a representative of each residue class of each point. The most commonly used example of a fundamental region is a period parallelogram, which is defined as

$$
P_{\Omega}=\left\{t \omega_{1}+s \omega_{2}: 0 \leq t, s<1\right\} .
$$

The generators of a lattice are by no means unique.

Proposition 2.2 ([8]). Two lattices $\Omega$ and $\Omega^{\prime}$ are equal if and only if

$$
\left(\begin{array}{l}
\omega_{1}^{\prime} \\
\omega_{2}^{\prime}
\end{array}\right)=\left(\begin{array}{ll}
a & b \\
c & d
\end{array}\right)\left(\begin{array}{l}
\omega_{1} \\
\omega_{2}
\end{array}\right)
$$

for some $\left(\begin{array}{ll}a & b \\ c & d\end{array}\right) \in S L_{2}(\mathbb{Z})$.

Remark 2.3. Given a lattice $\Omega$, one must choose generators $\omega_{1}$ and $\omega_{2}$ before a period parallelogram can be defined.

We say that two lattices are similar if $\Omega^{\prime}=\lambda \Omega$ for some $\lambda \in \mathbb{C}^{*}$.

Theorem 2.4 ([8]). Every lattice is similar to a lattice of the form $\langle 1, \tau\rangle$ for some unique $\tau \in T$, where

$$
T=\{z: \operatorname{Im}(z)>0,-1 / 2 \leq \operatorname{Re}(z)<1 / 2,|z| \geq 1(|z|>1 \text { when } \operatorname{Re}(z)>0)\} .
$$

To specific values of $\tau$ we attach names of similarity classes called shapes.

Definition 2.5. The following lattice shapes/collections of shape are defined as follows:

(1) A lattice $\Omega$ is square if $\tau(\Omega)=i$.

(2) A lattice $\Omega$ is triangular if $\tau(\Omega)=e^{2 \pi i / 3}$.

(3) A lattice $\Omega$ is rectangular if $\tau(\Omega)$ is pure imaginary.

(4) A lattice $\Omega$ is rhombic if $|\tau(\Omega)|=1$.

We now define elliptic functions on a given lattice. 
Definition 2.6. A meromorphic function, $f: \mathbb{C} \rightarrow \mathbb{C}_{\infty}$, is elliptic if there is a lattice $\Omega$ so that $f(z+\omega)=f(z)$ for all $\omega \in \Omega$.

The elliptic functions of Weierstrass are defined as the following convergent series:

$$
\wp_{\Omega}(z)=\frac{1}{z^{2}}+\sum_{\omega \in \Omega^{*}}\left(\frac{1}{(z-\omega)^{2}}-\frac{1}{\omega^{2}}\right)
$$

where $\Omega^{*}=\Omega \backslash\{0\}$.

By differentiating the series defining $\wp_{\Omega}$ we can obtain a series for $\wp_{\Omega}^{\prime}$ :

$$
\wp_{\Omega}^{\prime}(z)=-2 \sum_{\omega \in \Omega^{*}} \frac{1}{(z-\omega)^{3}} .
$$

When scaling the lattice we have the following relations, which we refer to as the homogeneity equations. For $k \in \mathbb{C}^{*}$ we have

$$
\wp_{k \Omega}(k z)=\frac{1}{k^{2}} \wp_{\Omega}(z)
$$

or, similarly,

$$
\wp_{k \Omega}(z)=\frac{1}{k^{2}} \wp_{\Omega}\left(\frac{z}{k}\right) .
$$

These relations follow directly from the definition of $\wp_{\Omega}$ and give a very useful tool for analyzing the families $\wp_{\lambda \Omega}$ where $\lambda \in \mathbb{C}^{*}$. The Weierstrass elliptic function $\wp_{\Omega}$ satisfies the following differential equation [8]:

$$
\left(\wp_{\Omega}^{\prime}(z)\right)^{2}=4 \wp_{\Omega}(z)^{3}-g_{2}(\Omega) \wp_{\lambda}(z)-g_{3}(\Omega),
$$

where $g_{2}$ and $g_{3}$ are invariants of the lattice. The invariants $g_{2}$ and $g_{3}$ can be written as follows:

$$
g_{2}(\Omega)=60 \sum_{\omega \in \Omega^{*}} \frac{1}{\omega^{4}} ; g_{3}(\Omega)=140 \sum_{\omega \in \Omega^{*}} \frac{1}{\omega^{6}},
$$

and so these invariants also have homogeneity properties:

$$
g_{2}(k \Omega)=\frac{1}{k^{4}} g_{2}(\Omega) ; g_{3}(k \Omega)=\frac{1}{k^{6}} g_{3}(\Omega) .
$$

These invariants also have the property that given complex numbers $g_{2}$ and $g_{3}$ with $g_{2}^{3}-27 g_{3}^{2} \neq 0$ there exists a unique lattice $\Omega$ so that $g_{2}=g_{2}(\Omega)$ and $g_{3}=g_{3}(\Omega)[8$.

Critical values of $\wp_{\Omega}$ correspond to zeros of $4 \wp_{\Omega}(z)^{3}-g_{2} \wp_{\lambda}(z)-g_{3}$. Using the Fundamental Theorem of Algebra we can write

$$
\left(\wp_{\Omega}^{\prime}(z)\right)^{2}=4\left(\wp_{\Omega}(z)-e_{1}\right)\left(\wp_{\Omega}(z)-e_{2}\right)\left(\wp_{\Omega}(z)-e_{3}\right) .
$$

It is known that the roots $\left\{e_{1}, e_{2}, e_{3}\right\}$ are distinct $\left[8\right.$ and are $e_{1}=\wp_{\Omega}\left(\omega_{1} / 2\right)$, $e_{2}=\wp_{\Omega}\left(\omega_{2} / 2\right)$, and $e_{3}=\wp_{\Omega}\left(\left(\omega_{1}+\omega_{2}\right) / 2\right)$.

The critical values satisfy important algebraic relations obtained by equating like terms in the factorization and the original expression $\left(\wp_{\Omega}^{\prime}(z)\right)^{2}=4 \wp_{\Omega}(z)^{3}-$ $g_{2} \wp_{\lambda}(z)-g_{3}$. We list the ones most relevant:

$$
e_{1}+e_{2}+e_{3}=0, \quad e_{1} e_{2}+e_{1} e_{3}+e_{2} e_{3}=\frac{-g_{2}}{4}, \quad e_{1} e_{2} e_{3}=\frac{g_{3}}{4} .
$$

Because of our focus on square lattices we develop in detail the formulas for the critical values in terms of the lattice. 
Proposition $2.7([8])$. Suppose that $\Omega$ is a square lattice. Then $e_{3}=0$ and $e_{2}=-e_{1}$. Furthermore, $g_{2}=4 e_{1}^{2}$ and $g_{3}=0$.

Certain lattices have symmetry about the real axis. These lattices are called real lattices.

Proposition 2.8. If $\Omega$ is a lattice so that $\bar{\Omega}=\Omega$, where $\bar{\Omega}=\{\bar{\omega}: \omega \in \Omega\}$, then

$$
\wp_{\Omega}: \mathbb{R} \backslash \Omega \rightarrow \mathbb{R}
$$

and

$$
\wp_{\Omega}^{\prime}: \mathbb{R} \backslash \Omega \rightarrow \mathbb{R}
$$

Proposition 2.9. Given a lattice $\Omega, \wp_{\Omega}$ is even.

Let $\mathbb{R}^{+}=\{t \in \mathbb{R}: t>0\}$. Then we have the following mapping property.

Proposition 2.10. If $\bar{\Omega}=\Omega$, then

$$
\wp_{\Omega}: \mathbb{R} \backslash \Omega \rightarrow \overline{\mathbb{R}^{+}} \text {. }
$$

An important benchmark quantity is defined as the following:

Definition 2.11. We define $\gamma>0$ as $\gamma^{2}=\wp_{\langle 1, i\rangle}(1 / 2)$.

This constant is referred to as the lemniscate constant; $\gamma \approx 2.62206 \ldots$, and an exact formula for $\gamma$ is the following:

$$
\gamma=\frac{1}{4} \sqrt{\frac{2}{\pi}}\left(\Gamma\left(\frac{1}{4}\right)\right)^{2},
$$

where $\Gamma(z)$ is the classical gamma function. Both the decimal approximation and the exact formula can be found in [1].

Using the homogeneity equations and lemniscate constant we can describe the critical values of $\wp_{\Omega}$ when $\Omega$ is square. Using (2.7) and the lemniscate constant $\gamma$, we have

$$
e_{1}=\wp\langle\lambda, \lambda i\rangle\left(\frac{\lambda}{2}\right)=\frac{1}{\lambda^{2}} \wp_{\langle 1, i\rangle}(1 / 2)=\frac{\gamma^{2}}{\lambda^{2}} .
$$

This gives the following.

Proposition 2.12. Let $\lambda \in \mathbb{C}^{*}$. The critical values of $\wp\langle\lambda, \lambda i\rangle$ are

$$
\left\{-e_{1}, 0, e_{1}\right\}=\left\{-\frac{\gamma^{2}}{\lambda^{2}}, 0, \frac{\gamma^{2}}{\lambda^{2}}\right\} \text {. }
$$

\section{The Fatou and Julia sets}

The notation for composition in this paper is $f^{\circ n}(z)=f^{n}(z)$ and does not mean $(f(z))^{n}$. Since a transcendental meromorphic function has an essential singularity at $\infty$ that cannot be extended, we need to carefully account for poles and points that arrive at poles in their forward obits. These points have finite forward orbits that terminate at infinity.

Definition 3.1. The Fatou set $F(f)$ of a meromorphic function $f: \mathbb{C} \rightarrow \mathbb{C}_{\infty}$ is defined to be

$$
F(f)=\left\{z:\left\{f^{n}\right\}_{n \in \mathbb{N}} \text { are defined and form a normal family at } z\right\} .
$$


Definition 3.2. The Julia set $J(f)$ of a meromorphic function $f$ is defined to be $J(f)=\mathbb{C}_{\infty} \backslash F(f)$.

The Fatou and Julia sets are forward and backward invariant [3, 4. Furthermore, the Fatou set is open, since it is a union of open sets, and so the Julia set is closed.

Theorem 3.3 ([3, Montel's Theorem]). Suppose that a family of functions $\mathcal{F}$ mapping $U \subset \mathbb{C}$ to $\mathbb{C}_{\infty}$ has the property that $f(U) \subset \mathbb{C}_{\infty} \backslash\{a, b, c\}$, where $a, b$ and $c$ are distinct points in $\mathbb{C}_{\infty}$. Then $\mathcal{F}$ is a normal family on $U$.

Using Montel's Theorem, we have the following fact. We say a set $X$ is completely invariant if $z \in X$ implies $f(z) \in X$ (unless $f(z)$ is undefined) and $f(w)=z$ implies $w \in X$.

Proposition 3.4 (4]). The Julia set is the smallest completely invariant closed set containing at least three points.

Since all lattice points are in the Julia set, the Julia set of an elliptic function is the closure of all the pre-images of infinity; i.e. $J(f)=\overline{O^{-}(\infty)}$.

The classification of Fatou components is an important tool for understanding the dynamics of both rational and transcendental meromorphic functions. This classification theorem is mostly due to Cremer [6] and Fatou [9, 10, and was first presented in this form by Baker, Kotus, and Lü [2].

Theorem 3.5 (4). Let $f$ be a meromorphic function on $\mathbb{C}$ and let $U$ be a periodic component of $F(f)$ of period $p$. Then we have exactly one of the following properties:

(1) $U$ contains an attacting periodic point $z_{0}$ of period $p$. Then $f^{n p}(z) \rightarrow z_{0}$ for $z \in U$ as $n \rightarrow \infty$, and $U$ is called the immediate basin of attraction for $z_{0}$.

(2) $\partial U$ contains a periodic point $z_{0}$ of period $p$ and $f^{n p}(z) \rightarrow z_{0}$ for $z \in U$ as $n \rightarrow \infty$. Then $\left(f^{p}\right)^{\prime}\left(z_{0}\right)=1$ if $z_{0} \in \mathbb{C}$. (For $z_{0}=\infty$ we have $\left(g^{p}\right)^{\prime}(0)=1$, where $g(z)=1 /(f(1 / z))$.) In this case, $U$ is a Leau domain (also called a parabolic domain).

(3) There exists an analytic homeomorphism $\phi: U \rightarrow \mathbb{D}$, where $D$ is the unit disk, such that $\left(\phi \circ f^{p} \circ \phi^{-1}\right)(z)=e^{2 \pi i \alpha} z$ for some $\alpha \in \mathbb{R} \backslash \mathbb{Q}$. In this case, $U$ is called a Siegel disk.

(4) There exists an analytic homeomorphism $\phi: U \rightarrow \mathbb{A}$ where $A$ is an annulus, and $A=\{z: 1<|z|<r\}$ for some $r>1$ such that $\left(\phi \circ f^{p} \circ \phi^{-1}\right)(z)=e^{2 \pi i \alpha} z$ for some $\alpha \in \mathbb{R} \backslash \mathbb{Q}$. In this case, $U$ is called a Herman ring.

(5) There exists $z_{0} \in \partial U$ such that $f^{n p}(z) \rightarrow z_{0}$ for $z \in U$ as $n \rightarrow \infty$, but $f^{p}\left(z_{0}\right)$ is not defined. In this case, $U$ is called a Baker domain.

The following result concerning elliptic functions was proved in [13].

Theorem 3.6. For any lattice $\Omega, \wp_{\Omega}$ has no cycle of Herman rings.

To rule out the existence of wandering domains and Baker domains we introduce the following family of functions.

Definition 3.7. A function is of class $S$ if it has finitely many critical values and finitely many asymptotic values.

Theorem 3.8 (4]). A meromorphic function of class $S$ has no wandering domains or Baker domains. 
It is the case that elliptic functions are of class $\mathrm{S}$ and therefore have no wandering domains or Baker domains. The following theorem follows from the fact that if a lattice $\Omega$ is square, then $\wp_{\Omega}$ has one free critical orbit.

Theorem $3.9([13])$. Let $\Omega$ be a square lattice. Then exactly one of the following must occur:

(1) $F\left(\wp_{\Omega}\right)$ is empty and $J(\wp \Omega)=\mathbb{C}_{\infty}$;

(2) $F\left(\wp_{\Omega}\right)$ is the basin of an attracting cycle;

(3) $F\left(\wp_{\Omega}\right)$ is the basin of a parabolic cycle;

(4) or $F\left(\wp_{\Omega}\right)$ consists of a cycle of Siegel disks and their pre-images.

\section{THE MAIN THEOREM}

Theorem 4.1. For each $\lambda \in \mathbb{C}^{*}$ the Julia set of $\wp_{\lambda}=\wp_{\langle\lambda, \lambda i\rangle}$ is connected.

The following theorem, adapted from [16, Theorem 3.1], is proved in [14. We present a similar proof here. From here on we will use the notation $\wp_{\lambda}=\wp_{\langle\lambda, \lambda i\rangle}$.

Theorem 4.2 ([14]). If every Fatou component of $F\left(\wp_{\Omega}\right)$ contains 0 or 1 critical values, then $J\left(\wp_{\Omega}\right)$ is connected.

Definition 4.3. Fix $\lambda \in \mathbb{C}^{*}$. Define $L_{1}=\left\{t \lambda^{-2}: t \in \mathbb{R}^{+}\right\}$and $L_{2}=-L_{1}$. Furthermore, define $S_{1}=\{t \lambda: t \in \mathbb{R} \backslash \mathbb{Z}\}$. Set $S_{2}=i S_{1}$.

Proposition 4.4. For each $\lambda \in \mathbb{C}^{*}$ we have that

$$
\wp_{\lambda}\left(S_{1}\right) \subset L_{1}
$$

and

$$
\wp_{\lambda}\left(S_{2}\right) \subset L_{2} .
$$

Proof. If $z \in S_{1}$, then $z=t \lambda$ for some $t \in \mathbb{R} \backslash \mathbb{Z}$. By the homogeneity equations (2.7), we have that

$$
\wp_{\lambda}(t \lambda)=\lambda^{-2} \wp_{1}(t)
$$

An elementary calculus exercise shows that $\wp_{1}(t) \geq \gamma^{2}$ for all $t \in \mathbb{R} \backslash \mathbb{Z}$ giving $\lambda^{-2} \wp_{1}(t) \in L_{1}$.

If $z \in S_{2}$, then $z=i t k$ for $t \in \mathbb{R} \backslash \mathbb{Z}$. We have that $i\langle\lambda, \lambda i\rangle=\langle\lambda, \lambda i\rangle$. Using the homogeneity equations a second time, we have

$$
\wp_{\lambda}(i t \lambda)=(i \lambda)^{-2} \wp_{1}(t)=-\lambda^{-2} \wp_{1}(t) .
$$

Since $\wp_{1}(t)>0$ for all $t \in \mathbb{R} \backslash \mathbb{Z}$, we have that $-\lambda^{-2} \wp_{1}(t) \in L_{2}$.

We observe that $e_{1} \in L_{1}$ and $e_{2} \in L_{2}$ by equation (2.14).

Lemma 4.5. Fix $\lambda \in \mathbb{C}^{*}$. Let $p \neq 0$. Then

$$
K(p)=\max \left\{\inf _{z \in L_{1}} d(z, p), \inf _{z \in L_{2}} d(z, p)\right\}>0,
$$

where $d$ is the Euclidean metric on $\mathbb{C}$.

The distance $K(p)$ depends on $\lambda$, but we write $K(\lambda, p)=K(p)$ as in Lemma 4.5.

Lemma 4.6. Let $C$ be a curve connecting $z_{1} \in L_{1}$ and $z_{2} \in L_{2}$ in $\mathbb{C}$. Let $H$ be a line passing through the origin. Then $C \cap H \neq \emptyset$. 
Proof. Parameterize $C$ on $[0,1]$ by $\gamma:[0,1] \rightarrow C$. If $C$ contains the origin, then we are done. Suppose that $C$ does not contain the origin. Let $\gamma(t)=r(t) e^{i \theta(t)}$. Let $\theta_{0}=\theta(0)$ and $\theta_{1}=\theta(1)=\theta_{0}+(2 k+1) \pi$, where $k \in \mathbb{Z}$. If $w \in H$, then $\arg (w)=\theta_{H}$ or $\arg (w)=\theta_{H}-\pi$ for some $\theta_{H} \in(0, \pi]$, and $H$ contains all such $w$ 's. We have that $\theta$ is a continuous function of $t$, so the Intermediate Value Theorem gives that $\theta\left(t_{0}\right)=\theta_{H}$ or $\theta\left(t_{0}\right)=\theta_{H}-\pi$, for some $t_{0} \in[0,1]$. Thus, there is $t_{0} \in[0,1] \mathrm{such}$ that $\gamma\left(t_{0}\right)=r\left(t_{0}\right) e^{i \theta\left(t_{0}\right)} \in H$.

We now prove the connectivity of $J\left(\wp_{\lambda}\right)$ in the case when $\wp_{\lambda}$ has an attracting or parabolic cycle. If $\wp_{\lambda}$ has an attracting or parabolic cycle $\left\{p_{0}, \ldots, p_{l-1}\right\}$, then $\wp_{\lambda}^{l n} \rightarrow p_{0}$ locally uniformly on the immediate basin of $p_{0}$ [4]. Furthermore, the immediate basin of an attracting or parabolic cycle contains a critical value [4.

Theorem 4.7. Suppose that $\wp_{\lambda}$ has an attracting or parabolic cycle $\left\{p_{0}, \ldots, p_{l-1}\right\}$. Then $J\left(\wp_{\lambda}\right)$ is connected.

Proof. It is sufficient to show that each Fatou component contains at most one critical value [14. The critical value 0 is not in the Fatou set, since it is a pole. We proceed by contradiction.

Suppose that a Fatou component $U$ contains both $e_{1}$ and $e_{2}=-e_{1}$. It is evident that $U$ must be the immediate basin of the cycle, since the immediate basin must contain a critical value 3, 4 and since $e_{1}$ and $-e_{1}$ are both in $U$. Each Fatou component is path connected, since it is open and connected. Let $C$ be a curve connecting $e_{1}$ and $-e_{1}$ in $U$.

All iterates of $\wp_{\lambda}$ are defined on $C$ since all pre-poles lie in the Julia set. Since $C$ is a compact subset of the immediate basin, $\left\{\wp_{\lambda}^{l n}\right\}_{n \in \mathbb{N}}$ converges uniformly on $C$ to the constant function $p_{0}$ in the Euclidean metric [3, 4. This means that for all $\epsilon>0$ there is an $N \in \mathbb{N}$ so that $\sup _{z \in C}\left|\wp_{\lambda}^{l n}(z)-p_{0}\right|<\epsilon$ for $n>N$.

We claim that for each $m \in \mathbb{N}, \wp_{\lambda}^{m}(C)$ contains a point on each of $L_{1}, L_{2}, S_{1}$, and $S_{2}$ and is connected. We proceed by induction. We first establish these properties for the $m=0$ case, $C$.

By construction $C$ contains a point on $L_{1}$ and on $L_{2}$. We establish that $C \cap S_{i} \neq \emptyset$ for $i=1,2$. Fix $i$. Now $\overline{S_{i}}$ is a line passing through the origin, so by Lemma 4.6 we have that $C \cap \overline{S_{i}} \neq \emptyset$. Since $C$ does not contain any poles, we have that $C \cap S_{i} \neq \emptyset$.

For the induction hypothesis, we assume that $\wp_{\lambda}^{m}(C)$ contains a point in $L_{1}$, $L_{2}, S_{1}$, and $S_{2}$ and is connected. We can immediately establish that $\wp_{\lambda}^{m+1}(C)$ is connected, since all iterates are defined on $C$ and $\wp_{\lambda}$ is continuous off its pre-poles. Furthermore, $\wp_{\lambda}^{m+1}(C)$ contains a point on $L_{1}$ and a point on $L_{2}$ by Proposition 4.4. Lastly, we can establish that $\wp_{\lambda}^{m+1}(C)$ contains a point on $S_{1}$ and $S_{2}$ by Lemma 4.6.

If $p_{0} \notin L_{2}$, choose $a_{n}$ in $\wp_{\lambda}^{\ln }(C) \cap L_{2}$ for each $n \in \mathbb{N}$ (if $p_{0} \in L_{2}$, then choose $a_{n}$ in $L_{1}$ ). We have that

$$
\sup _{z \in C}\left|\wp_{\lambda}^{l n}(z)-p_{0}\right| \geq\left|a_{n}-p_{0}\right|>K\left(p_{0}\right)>0
$$

for all $n \in \mathbb{N}$ by Lemma 4.5. This contradicts the uniform convergence of $\left\{\wp_{\lambda}^{l n}\right\}_{n \in \mathbb{N}}$ on $C$ to the constant function $p_{0}$. Thus every Fatou component contains at most one critical value, and so the Julia set is connected.

We now prove the main result for all square lattices. 
Proof of Theorem 4.1. If $F(\wp)$ is empty, then $J(\wp)=\mathbb{C}_{\infty}$ and so is connected. (For example, this occurs when the lattice is a so-called "rhombic square" lattice [11. It also occurs when the critical orbit lands on a pole. There are other examples as well; n.b. [12]). If $\wp$ has an attracting cycle or a parabolic cycle, we have that $J(\wp)$ is connected by Theorem 4.7. If the Fatou set consists of pre-periodic Siegel disks, then the Julia set is connected [4, 14. This exhausts all possible Fatou components. Thus $J\left(\wp_{\lambda}\right)$ is connected for all $\lambda \neq 0$.

\section{RELATED RATiOnAL MAPS}

In this section we begin exploring connections between higher degree rational maps and Weierstrass elliptic functions on square lattices. The main result of this chapter is Theorem 5.6. This section is motivated by work on singularly perturbed maps by Bob Devaney et al. In 7] B. Devaney, D. Look, and D. Uminsky describe an escape trichotomy similar to the escape dichotomy for the quadratic map. In particular, they prove that if the free critical orbit remains bounded, then the Julia set is connected. Using the techniques developed in the previous section we present a proof of this result for a conformally conjugate family of rational maps.

Proposition 5.1 ([1]). For the $\left\{g_{2}, g_{3}\right\}$ invariant parameterization $\wp_{\left\{g_{2}, g_{3}\right\}}$ of the Weierstrass elliptic functions we have the following Laurent series centered at zero:

$$
\wp_{\left\{g_{2}, g_{3}\right\}}(z)=\frac{1}{z^{2}}+\sum_{k=2}^{\infty} c_{k} z^{2 k-2},
$$

where $c_{2}=g_{2} / 20, c_{3}=g_{3} / 28$, and

$$
c_{k}=\frac{3}{(2 k+1)(k-3)} \sum_{m=2}^{k-2} c_{m} c_{k-m}
$$

for $k>3$. This series converges on $0<|z|<R$, where $R=\min _{\omega \in \Omega^{*}}|\omega|$.

Our family of interest is the similarity class of square lattices. Thus, we have that $g_{3}=0$ and $g_{2}=4 \gamma^{4} / \lambda^{4}$, where $\lambda$ generates the lattice $\langle\lambda, \lambda i\rangle$. This gives the following.

Proposition 5.2 ([1]). For $\wp_{\lambda}$ we have the following Laurent series centered at zero:

$$
\wp_{\lambda}(z)=\frac{1}{z^{2}}+\sum_{k=1}^{\infty} c_{2 k} z^{4 k-2}=\frac{1}{z^{2}}+\frac{\gamma^{4}}{5 \lambda^{4}} z^{2}+\cdots,
$$

where $c_{2}=\gamma^{4} 5 \lambda^{4}$ and

$$
c_{2 k}=\frac{3}{(4 k+1)(2 k-3)} \sum_{m=1}^{k-1} c_{2 m} c_{2(k-m)}
$$

for $k>1$. This series converges on $0<|z|<|\lambda|$.

By truncating the infinite Laurent series we obtain parameterized families of rational approximations to $\wp_{\lambda}$. We define the following family:

$$
P_{\lambda}(z)=\frac{1}{z^{2}}+\frac{\gamma^{4}}{5 \lambda^{4}} z^{2},
$$


since

$$
\wp_{\lambda}(z)=P_{\lambda}(z)+O\left(\frac{z^{6}}{\lambda^{8}}\right)
$$

from Proposition 5.2 .

Just as with $\wp_{\lambda}, P_{\lambda}$ satisfies a homogeneity equation:

$$
P_{\lambda}(\lambda z)=\frac{1}{\lambda^{2}} P_{1}(z)
$$

This family also has similar mapping properties.

Definition 5.3. Fix $\lambda \in \mathbb{C}^{*}$. Define

$$
L_{1}=\left\{t \lambda^{-2}: t \in \mathbb{R}^{+}\right\}
$$

and

Furthermore, define

$$
L_{2}=-L_{1}
$$

Set

$$
\tilde{S}_{1}=\{t \lambda: t \in \mathbb{R} \backslash\{0\}\}
$$

$$
\tilde{S}_{2}=i \tilde{S}_{1}
$$

Proposition 5.4. For $\lambda \in \mathbb{C}^{*}$ we have that $P_{\lambda}\left(\tilde{S}_{1}\right) \subset L_{1}$ and $P_{\lambda}\left(\tilde{S}_{2}\right) \subset L_{2}$.

Proof. If $z \in \tilde{S}_{1}$, then $z=t \lambda$ for some $t \in \mathbb{R} \backslash\{0\}$. By the homogeneity equations (5.6), we have that

$$
P_{\lambda}(t \lambda)=\lambda^{-2} P_{1}(t) .
$$

Furthermore, $P_{1}(t)>0$ for all $t \in \mathbb{R} \backslash\{0\}$, giving the fact that $\lambda^{-2} P_{1}(t) \in L_{1}$.

If $z \in S_{2}$, then $z=i t k$ for $t \in \mathbb{R} \backslash\{0\}$. We have that $P_{i \lambda}=P_{\lambda}$. Using the homogeneity equations a second time, we have

$$
P_{\lambda}(i t \lambda)=P_{i \lambda}(i t \lambda)=(i \lambda)^{-2} P_{1}(t)=-\lambda^{-2} P_{1}(t) .
$$

Since $P_{1}(t)>0$ for all $t \in \mathbb{R} \backslash\{0\}$, we have that $-\lambda^{-2} P_{1}(t) \in L_{2}$.

The critical points of $P_{\lambda}$ are $\left\{z: z^{4}=\frac{5 \lambda^{4}}{\gamma^{4}}\right\}$ and the point at infinity. The finite critical points can be written as $i^{k} \frac{\sqrt[4]{5} \lambda}{\gamma}$, where $k=0,1,2$, or 3 . The point at infinity is a super-attracting fixed point. This leaves one free critical orbit, since after two iterations all critical points (except infinity) land on the same point. Thus $P_{\lambda}$ can have at most one finite non-repelling periodic cycle [3].

Theorem 5.5. Suppose that $P_{\lambda}$ has a finite attracting or parabolic cycle. Then each Fatou component contains at most one critical value.

Proof. This proof proceeds identically to the proof of Theorem 4.7

We present the following theorem.

Theorem 5.6. Suppose that $P_{\lambda}$ has an attracting cycle, parabolic cycle, cycle of Siegel disks, or a strictly pre-periodic critical value. Then $J\left(P_{\lambda}\right)$ is connected.

Proof. If $P_{\lambda}$ has an attracting cycle or parabolic cycle, then Theorem 4.7 gives that each Fatou component contains at most one critical value. If $P_{\lambda}$ has a cycle of Siegel disks or a strictly pre-periodic critical value, then the critical values are contained in the Julia set, and so again, each Fatou component contains at most one critical value. By applying [16, Theorem 3.1] we have the result. 


\section{REFERENCES}

1. Milton Abramowitz and Irene A. Stegun (eds.), Handbook of Mathematical Functions with Formulas, Graphs, and Mathematical Tables, Dover Publications Inc., New York, 1992. Reprint of the 1972 edition. MR 1225604 (94b:00012)

2. I. N. Baker, J. Kotus, and Lü Yinian, Iterates of meromorphic functions. I, Ergodic Theory Dynam. Systems 11 (1991), no. 2, 241-248. MR1116639 (92m:58113)

3. Alan F. Beardon, Iteration of Rational Functions, Complex analytic dynamical systems, Graduate Texts in Mathematics, vol. 132, Springer-Verlag, New York, 1991. MR1128089 (92j:30026)

4. Walter Bergweiler, Iteration of meromorphic functions, Bull. Amer. Math. Soc. (N.S.) 29 (1993), no. 2, 151-188. MR1216719 (94c:30033)

5. Joshua Clemons, Dynamical properties of Weierstrass elliptic functions on square lattices, University of North Carolina at Chapel Hill (2010), Ph.D. dissertation.

6. H. Cremer, Über die schrödersche funktionalgleichung und das schwarzsche eckenabbildungsproblem, Ber. Verh. Sächs. Akad. Wiss. Leipzig, Math-Phys. Kl. 84 (1932), 291-324.

7. Robert L. Devaney, Daniel M. Look, and David Uminsky, The escape trichotomy for singularly perturbed rational maps, Indiana Univ. Math. J. 54 (2005), no. 6, 1621-1634. MR2189680 (2006i:37105)

8. Patrick Du Val, Elliptic Functions and Elliptic Curves, London Mathematical Society Lecture Note Series, No. 9, Cambridge University Press, London, 1973. MR0379512 (52:417)

9. P. Fatou, Sur les équations fonctionnelles, Bull. Soc. Math. France 47 (1919), 161-271. MR 1504787

10. Sur les équations fonctionnelles, Bull. Soc. Math. France 48 (1920), 33-94, 208-314. MR 1504797

11. Jane Hawkins, Smooth Julia sets of elliptic functions for square rhombic lattices, Topology Proc. 30 (2006), no. 1, 265-278, Spring Topology and Dynamical Systems Conference. MR 2280672 (2007j:37076)

12. Jane Hawkins and Lorelei Koss, Ergodic properties and Julia sets of Weierstrass elliptic functions, Monatsh. Math. 137 (2002), no. 4, 273-300. MR.1947915 (2003j:37066)

13. , Parametrized dynamics of the Weierstrass elliptic function, Conform. Geom. Dyn. 8 (2004), 1-35 (electronic). MR2060376 (2005b:37089)

14. $\ldots$ Connectivity properties of Julia sets of Weierstrass elliptic functions, Topology Appl. 152 (2005), no. 1-2, 107-137. MR2160809 (2007b:37098)

15. Jane M. Hawkins and Daniel M. Look, Locally Sierpinski Julia sets of Weierstrass elliptic $\wp$ functions, Internat. J. Bifur. Chaos Appl. Sci. Engrg. 16 (2006), no. 5, 1505-1520. MR2254870 (2007h:37068)

16. John Milnor, On rational maps with two critical points, Experiment. Math. 9 (2000), no. 4, 481-522. MR1806289 (2001k:37074)

Department of Mathematics, Phillips Hall CB\#3250, University of North Carolina at Chapel Hill, Chapel Hill, North Carolina 27599-3250

E-mail address: jclemons@vt.edu 